\title{
Research on the Influence of Network Public Opinion on Sports Management
}

\author{
Yan Gong ${ }^{1, a}$ \\ ${ }^{1}$ Jiangxi Science and Technology Normal University, Nanchang, Jiangxi, China, 330003 \\ ${ }^{a}$ email,
}

Keywords: Network Public Opinion Analysis; Sports Management Work; Impact Analysis

\begin{abstract}
With the continuous development of the computer, the network has been widely used and widely used in the world and the network has become an indispensable part of the life and work of the masses. Network has become the place of ideological exchange and it creates a global network world in the real world, where the local culture, goods combine together to form a huge platform for the exchange. Network discussed and released other people's works, published words and deeds, and then formed a group offensive, and ultimately form the network public opinion. Therefore, the research on the network public opinion has become the subject of government departments. Through the analysis of the network public opinion, we explore the impact of network public opinion on sports management.
\end{abstract}

\section{Introduction}

In today's society, the network has become a part of people's lives and people enjoy the convenience of the network at the same time, also affected by the network. The network provides a huge platform for communication between people in this platform can be equal to express their position and perspective. In recent years, the network public opinion, social public opinion and so become social-oriented attention caused by the relevant departments, in early 2012, the number of Chinese Internet users reached 593 million people, and the use of micro-blogging there are 330 million people, indicating that Chinese rapid development of the Internet, Coverage of the region and the crowd rising trend is amazing, network news and information transmission channels and forms of increasingly rich. Sports public opinion, the Chinese football reform, Zhang Shangwu events become the focus of attention to sports news, the parties or sports management departments have formed a major blow, how to correctly handle the network public opinion has become the main problem faced by sports managers.

\section{The Network Public Opinion Analysis}

Chinese research on the network public opinion is relatively late, and even now do not have accurate positioning of public opinion. Network public opinion is an extension of the virtual network, can be summarized as a social group through a network of personal events on a stand. Network public opinion by the high quality of people's favorite, especially the current college students, we can say that college students have become the main force of social public opinion, college students often through the network to obtain information at the same time, to express their own ideas [1].

Network public opinion is part of society, is the masses of public events held by the attitude, willingness, mood and thought, and so the performance of the collection. As a center of network public opinion, college students often focus on their own point of view, predict and judge the direction of the development of things, political, economic, cultural and other areas of the high degree of concern, they dare to expose the dark side of society and society unknown shadow. This topic is easy to form a certain public opinion, and stir up the reaction of the masses led to a certain degree of social unrest. As the network of high-speed, large data and other characteristics, the masses of the first event to see the world's big news, for a quick discussion of an event, the formation of a certain topic, if some extreme people in the understanding of some social dark Time, 
and even there will be some extreme behavior. For example: the Diaoyu Islands incident, the Chinese patriotic youth rushed into the store on the Japanese products.

Through the above analysis, we can know the main body of the Internet public opinion is Internet users, Internet users in the Internet platform to express their views, with their own unique perspective, participation and exchange, and ultimately the formation of views flow. Network public opinion is a double-edged sword, on the one hand can help people participate in politics, to help the country to understand the reaction of the masses of the national events, this time the public opinion is public opinion. On the other hand, false news and corruption messages not only hurt the interests of individual masses, but also cause public outrage, seriously disrupting the order of the network.

\section{The Sports Public Opinion Analysis}

The Characteristics of Sports Public Opinion. With the deepening of the network public opinion, found that the network of public opinion on the political, cultural, economic aspects are involved. This article on the sports network public opinion, in-depth study to understand the sports public opinion on the development of sports and management has how the impact.

Sports as a key topic of concern to our people, the natural existence of most of the video on the network, pictures, especially in the Olympic Games, the Asian Games, the World Cup and NBA time, sports is a hot topic can be used directly from the micro-blogging hot to intuitively reflect the focus of attention of the masses [2].

Sports public opinion in different sports in the embodiment is also significantly different, such as the NBA, the World Cup as the world's impact of the game and the topic directly to become the center of sports public opinion, and some gymnastics, hockey and other sports in the country few people pay attention and attention, making sports Public opinion in different projects appear hot and popular difference. Sports public opinion will appear a certain point of view and this point is not a particular event, but personal. The masses will be very good athletes have high expectations, once the athletes appear state slip and play disorders, social public opinion will be a certain blow to it, then the manager must manage the hearts of athletes.

The Status of Sports Public Opinion. With the continuous popularization of network technology, the continuous development of social civilization, micro-blogging from birth to later development has become the focus of our network of public opinion. Micro-blogging builds a network platform to promote the user and the user to form a relationship between the concern, and throw hot topics lead to hot. Some of the users of the fans amazing number of these words and deeds will be spread to tens of thousands of fans and this transmission is very influential, faster than the traditional paper media spread, more efficient. Sports micro-blogging timely update the situation of athletes and fans to form a certain interaction can be very good to promote the development of sports. The views of the fans can also be faster statistics, fans in the form of text or digital hair on the network, so in the statistical and absorption point of view also provides a convenient.

\section{The Impact of the Network Public Opinion on the Social Sports Management}

Network public opinion has been involved in various fields, to a certain extent, Chinese sports management work have a certain impact. Network public opinion through public opinion pressure, forcing sports managers to stop a series of management measures for the management of sports managers in China has brought great challenges. At the same time, the network public opinion can also help the sports industry better development, to accept the disease absorption network of public opinion can help us to establish a sound sports system.

Promote the Sports Technology Construction Tends to Improve. Internet public opinion can effectively help the publicization of sports equipment, in order to build a well-off society, the whole people to promote the initiative proposed by the government. Public sports infrastructure is an important step in the development and planning of our country. Public sports service has become the main function of the government, including public sports facilities construction, public sports 
education service, national physical examination and so on. Government departments and management departments in the actual practice process will be flawed, or wrong, this time you can through the network public opinion for the first collection of information collection, for some constructive comments in order to arrange the following to do things.

Analysis of network public opinion to understand the public's sports needs, to promote the functional departments to grasp the public's views on the construction of public sports assistance, combined with the actual situation, the relevant control. Local governments can access information through local networks and promote the construction of public sports facilities with distinctive functional areas that have distinctive characteristics. Official micro-operators should always pay attention to the cultural online sports public opinion oriented, establish a true public opinion-oriented. With the cultural quality of Internet users in China is getting higher and higher, the network public opinion is not just a direction, a lot of public opinion from the various aspects of the incident evaluation, and dig behind the "hidden". For example: "Liu Xiang retired" this topic, most people expressed their understanding of Liu Xiang's retirement, for the behavior of Internet users also have different views. Network is now watching sports events are generally broadcast through the network, for example, Sina's NBA live, etc., because the game can be watched at the same time can communicate and comment, making the fans very happy. Similarly, the management of the evaluation of the players through the players can see the fans of each player's favorite degree, but also in the supervision of the whole Internet users, if there is a clear foul and the referee did not notice that Internet users can form this action public opinion. Network public opinion in a certain extent for the relevant government departments in China has made a great contribution to the management [3].

Promote the Development of Sports Goods In China. Network in the contemporary environment is not only the largest information platform, but also the largest trading platform. Taobao, Lynx and other Internet trading platform will be the market into the Internet, the formation of a no boundaries of the market. With the continuous development of sports information, more and more people concerned about sports events, the network to develop all kinds of sports club live events also increased sports consumption. Sports business needs of the network sports opinion to conduct a precise analysis in order to seize the pain of the masses of the masses in order to help the masses to better carry out commodity consumption.

Sports sales model can be through the network channel, the network market has become a huge cake, online sports goods and sports services are more and more talk. Sports business through the network of public opinion to understand the market demand and hot topics, coupled with the online collection of various manufacturers of useful information, a reasonable purchase and shipping, to maximize the benefits. With the table tennis distance, the different areas of the table tennis goods eye different, and some areas need the brand racket feel, and some are the quality, as well as the quality and severity of table tennis have different requirements. Through the local table tennis related forum or micro-blogging to understand the brand of table tennis most popular, and then the manufacturers of the relevant selection, through online screening, offline understanding, selected manufacturers, the rational use of resources in order to achieve the benefits of maximize. The network also has the corresponding customer resources, network sports public opinion users are mostly sports enthusiasts, this is conducive to business development of customers, compared to the traditional way of selling marketing, this way more timeliness, extensive.

\section{Conclusion}

The development time of network public opinion analysis in Chinese is not long, which involves a number of areas of cultural exchanges, and which also includes real-time monitoring of hot spots, network sensitive information, marketing, etc., and it can also get good use in sports management. Sports network public opinion gap can set sports management as a public opinion center to guide the masses of sports concern, and through the corresponding network platform and the masses interact, which is the agreement of sports development and people. Public supervision can effectively guarantee the fairness of sports competition, while the network platform for public 
opinion analysis can help marketing sports-related products, improve the sales of sports industry and make the enterprise develop [4].

\section{References}

[1] Fu Yaping, Zhang Wei. Study on hot news events of public opinion in the perspective of public management - based on the empirical analysis of "top ten influential litigation" in recent five years [J]. E-Government, 2011, 11: 16-22.

[2] Guo Mingfei, Chen Lanlan. Network space ideological security situation and strategy - based on large data background and analysis[J]. Jianghan Forum, 2016, 05: 28-32.

[3] Wu Jie. On the network public opinion to build a harmonious and beautiful campus network culture environment strategy analysis[J]. Information construction, 2016, 04: 82

[4] Chen Bikun, Wang Yuefen, Liao Haihan. Study on the current situation and development trend of social public opinion analysis and decision support in big data age [J]. Information Science, 2016, 10: 8-14. 